\title{
Robust Highly Emissive Probe For Plasma Potential Measurements In The Edge Region Of Toroidal Plasmas
}

\author{
B.S. Schneider ${ }^{* a}$, S. Costea ${ }^{a}$, C. Ionita ${ }^{a}$, R. Schrittwieser ${ }^{a}$, V. Naulin ${ }^{b}$, J.J. \\ Rasmussen $^{b}$, R. Stärz ${ }^{a c}$, N. Vianello ${ }^{d}$, J. Kovacic ${ }^{e}$ and T. Gyergyek ${ }^{f}$ \\ ${ }^{a}$ Institute for Ion Physics and Applied Physics, University of Innsbruck \\ Technikerstr. 25, 6020 Innsbruck, Austria \\ ${ }^{b}$ DTU Physics, Technical University of Denmark \\ Anker Engelunds Vej 1, 2800 Kgs. Lyngby, Denmark \\ ${ }^{c}$ Mechatronic Department, Management Center Innsbruck \\ Universitätsstraße 15, 6020 Innsbruck, Austria \\ ${ }^{d}$ Centre de Recherches en Physique des Plasmas, Ecole Polytechnique Fédérale de Lausanne \\ CH-1015 Lausanne, Switzerland \\ e Jozef Stefan Institute \\ Jamova cesta 39, 1000 Ljubljana, Slovenia \\ ${ }^{f}$ Faculty of Electrical Engineering, University of Ljubljana \\ Trzaska 25, 1000 Ljubljana Slovenia \\ E-mail: bernd.s.schneider@uibk.ac.at, stefan.costea@uibk.ac.at, \\ codrina.ionita@uibk.ac.at, roman.schrittwieser@uibk.ac.at, \\ vona@fysik.dtu.dk, jjra@fysik.dtu.dk, Ronald.Staerz@mci.edu, \\ nicola.vianellodepfl.ch, jernej.kovacicaijs.si, \\ tomaz.gyergyekeijs.si
}

In this work we present results of a newly developed highly emissive Langmuir probe with $\mathrm{LaB}_{6}$, $\mathrm{TiC}$ and pure carbon probe tips for the use in high temperature plasmas and dense plasmas. The work function, the main parameters for electron emission, of $\mathrm{LaB}_{6}$ is $2,7 \mathrm{eV}$, that of $\mathrm{TiC} 3,4 \mathrm{eV}$, and 4,6 eV for C. Emissive electrical probes have the advantage of measuring the plasma potential directly with high temporal resolution at the position of the probe. First measurements are carried out in a Double Plasma Machine with 1,5 mm of diameter probe tip for $\mathrm{LaB}_{6}$ and $1 \mathrm{~mm}$ for $\mathrm{TiC}$ and $\mathrm{C}$.

First EPs Conference on Plasma Diagnostics - 1st ECPD,

14-17 April 2015

Villa Mondragone, Frascati (Rome) Italy

* Speaker. 


\section{Introduction}

Electron-Emissive Probes (EEP) have first been mentioned in 1923 by Noble prize winner Irving Langmuir [1]. He also first described how to determine the plasma potential from the currentvoltage $(I-V)$ characteristic of a cold probe. Since then, a great variety of probes, utilizing various measuring techniques in all kinds of plasmas, have been developed, with the research and development continuing also today.

At high electron emission EEPs float on or near plasma potential, thus allowing to display this highly important plasma parameter directly at the position of the probe with temporal resolution limited practically only by the data acquisition system. With a cold probe, the plasma potential can be determined only indirectly, either from its floating potential, provided the electron temperature is known too, or from the electron current "knee" of its $I-V$ characteristic [2-6].

Here we present plasma potential measurements by a newly developed highly emissive EEPs. The results obtained from floating and inflection point analysis are compared with "knee" method of cold probe $I-V$ characteristics [2-7]. With sufficient heating of the probe pin, the EEP's floating potential does indeed become more positive to eventually reach a value which agrees very well with the values of the plasma potential determined by the above-mentioned methods [7-13]. Having a probe head with several suitably arranged emissive probes, also electrical fields and their fluctuations can be determined $[9,11,13,14]$. Electric field fluctuations are directly related to cross-field particle transport in tokamaks and stellarators, which can affect plasma confinement.

\section{Probe Design}

Prototypes of EEPs have been developed using an 1,5 mm diameter pin for $\mathrm{LaB}_{6}$ with a total length of $10 \mathrm{~mm}$ and $1 \mathrm{~mm}$ diameter pins for TiC-covered carbon and pure carbon with total lengths of $30 \mathrm{~mm}$ (see Fig.1). The work function of $\mathrm{LaB}_{6}$ is $2,7 \mathrm{eV}$ [15], that of $\mathrm{TiC} 3,4 \mathrm{eV}$ [15] and carbon's work function is $4,6 \mathrm{eV}$ [16]. The melting points of these three materials are $2482 \mathrm{~K}$ [16] $\left(\mathrm{LaB}_{6}\right), 3430 \mathrm{~K}$ (TiC) [18] and $4023 \mathrm{~K}(\mathrm{C})$ [19]. Although carbon has the highest heat resistance, its high work function demands much higher temperatures to emit an electron current density equal to that emitted by $\mathrm{LaB}_{6}$ or $\mathrm{TiC}$ when heated. The EEP with $\mathrm{LaB}_{6} \operatorname{rod}(\mathrm{LaBP})$ is electrically heated through a carbon ring contact in the front and a Mo cylindrical pin holder at the rear end of the rod. The front carbon contact ring is made of one of the few materials that sufficiently resists the heat load during the total measurement time without risk of oxidizing or melting.
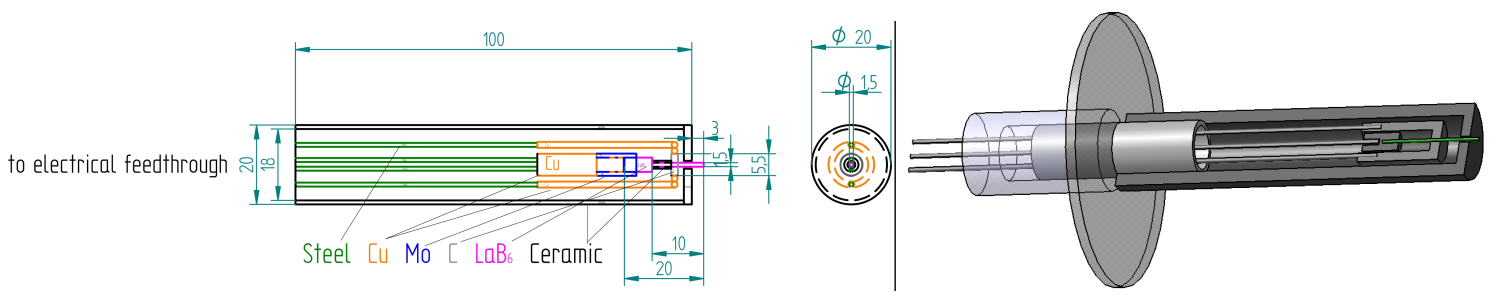

Figure 1: A sketch of the emissive probe design for the $\mathrm{LaB}_{6}$ (left) and the $\mathrm{TiC}$ and pure $\mathrm{C}$ probe (right). The heating current flows directly through the rear part of the rods and and the resulting heat is conducted to its front facing the plasma. 
The rods are all heated only on a part of their lengths. The actual probe tips protruding $3 \mathrm{~mm}$ into the argon plasma, are heated by conduction. For the emissive $\mathrm{TiC}$ and $\mathrm{C}$ probe (TiCP and $\mathrm{CP}$ ) the rear Mo holder of the $\mathrm{LaB}_{6}$ probe has been exchanged by a graphite holder to resist even higher temperatures. The front contact ring has been pushed further to the tips of the pins for more stable operation. The outer shielding material of the probe has been exchanged by graphite instead of $\mathrm{Al}_{2} \mathrm{O}_{3}$ massively reducing $\mathrm{O}_{2}$ poisoning of the plasma, affecting the floating and plasma potential, when the probe is heated.

\section{Experimental device}

The argon plasma is produced by a gas discharge in a $900 \mathrm{~mm}$ long, $440 \mathrm{~mm}$ diameter cylindrical vacuum chamber, the Innsbruck DPM (Double-Plasma Machine). Two negatively biased $0,15 \mathrm{~mm}$ diameter tungsten filaments at the upper part of the chamber are heated $\left(I_{\text {heat }, \text { fil } 1,2}\right)$ by two power supplies as sketched in Fig. 2 to produce an accordingly stable, almost homogeneous plasma with a discharge current $I_{\text {discharge } 1,2}$ from the filaments to the chamber wall. The achievable plasma density in the chamber lies between $10^{14}$ and $5 \cdot 10^{17} \mathrm{~m}^{-3}$ with an electron temperature $T_{e}$ ranging between 0,5 and $2 \mathrm{eV}$. The probe tip temperatures of the EEPs to reach the plasma potential while floating, compensating the incoming electron density arriving from the plasma, are between $T_{W, L a B 6}=1250$ and $1750 \mathrm{~K}$ for $\mathrm{LaB}_{6}, T_{W, T i C}=1320$ to $1800 \mathrm{~K}$ for TiC and $T_{W, C}=2000$ to $2500 \mathrm{~K}$ for $\mathrm{C}$. The emissive probes are mounted on the axis of the vacuum cylinder at one end

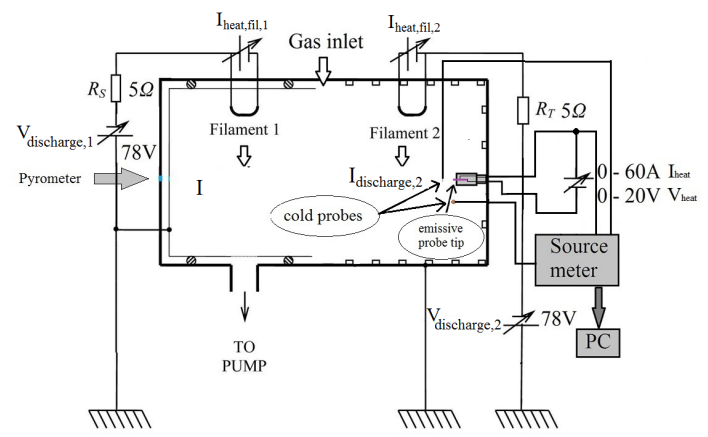

Figure 2: Experimental setup for probe measurements in the DPM.

of the chamber reaching $100 \mathrm{~mm}$ into the plasma (see Fig. 2). A 60A-20V power supply ensures the necessary power to heat the emissive probe tips to the necessary temperatures. A computer controlled source meter is used to sweep the EEPs, as well as two cold Langmuir probes and to record $I-V$ characteristics. As cold probes a thin $5 \mathrm{~mm}$ diameter brass disk, which is mounted about $50 \mathrm{~mm}$ underneath of the emissive probe tip and a $0,15 \mathrm{~mm}$ diameter, $3 \mathrm{~mm}$ long cylindrical tungsten probe, mounted $40 \mathrm{~mm}$ directly in front of the emissive probe tip are used. A pyrometer is aimed on the probe tip from outside the chamber through a quartz window, recording temperatures between $1263 \mathrm{~K}$ and $3273 \mathrm{~K}$. For the probe testing the plasma density $n_{p l}$ was ranging between $3,5 \cdot 10^{14}$ and $4,5 \cdot 10^{14} \mathrm{~m}^{-3}$ with an electron temperature alternating slowly between 0,7 and $1,5 \mathrm{eV}$, set by an argon pressure of $2 \cdot 10^{-3}$ mbar and a discharge current $I_{\text {discharge } 1,2}=200 \mathrm{~mA}\left(V_{\text {discharge } 1,2}\right.$ $=78 \mathrm{~V}$ ) for each $\mathrm{W}$ filament. The floating potential $V_{f l}$ of the plasma ranges under these settings 
typically varies between -12 and $-3 \mathrm{~V}$. The reason for the different floating and plasma potential values during an experiment are not completely clear.

\section{Results and discussion}

The selected probe characteristics presented in Fig. 3 have been obtained with the $\mathrm{LaB}_{6}$ and the TiC probe. Both characteristics show the typical behaviour of emissive probes [1,7-12]: The emission current appears on the negative side of the characteristics superimposed on the ion saturation current, with increasing magnitude when the heating is raised. The electron saturation current of the probes, which should in principle remain unaffected by the electron emission, also shows a strong increase. Such effects have been seen earlier by emissive probes, but the reasons are not yet
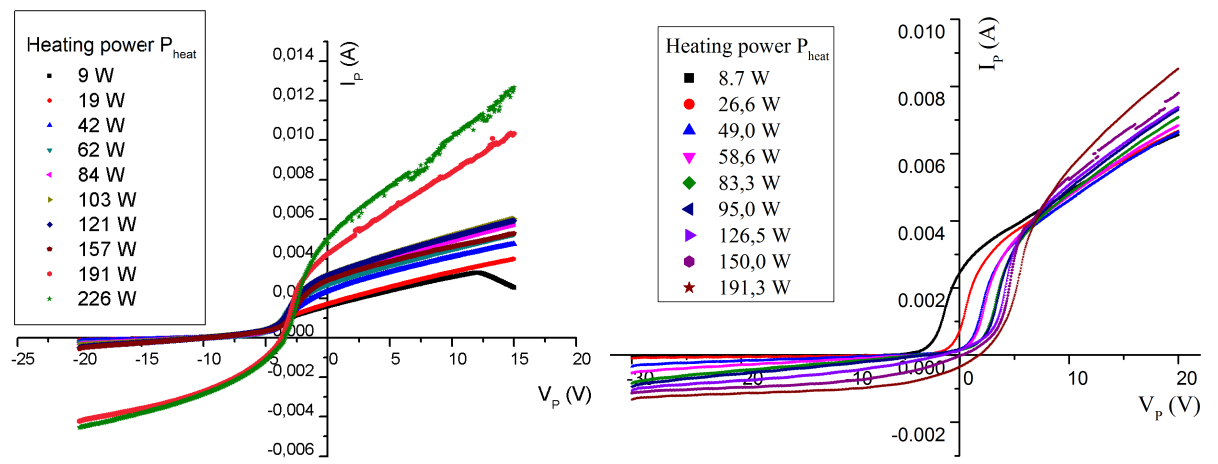

Figure 3: $I-V$ characteristics of the $\mathrm{LaB}_{6}$ (left panel) and $\mathrm{TiC}$ (right panel) probe recorded at different heating powers. A re-increasing of the resistivity of the $\mathrm{LaB}_{6}$ probe pin at a heating power of $163 \mathrm{~W}$ leads to a fast increase of the electron emission.

clear [20,21]. On the other hand, this effect does not change the principle property that the floating potential of the EEP is found very close to the plasma potential. Estimates show that in particular with $\mathrm{TiC}$, with one of the almost highest reachable total electron emission currents of all know materials (over a factor of three times higher than $\mathrm{LaB}_{6}$ ), the average electron current density in high temperature and dense plasmas can be compensated by the electron emission current density, which is a prerequisite for the emissive probe to float on the plasma potential.

The behaviour of the floating potential of the EEPs when heated is represented in Fig. 4 for all three probes. The error bars in Fig. 4 and Fig. 5 represent a voltage drop of $V_{\text {heat }} / 5$, where $V_{\text {heat }}$ is the heating voltage of the probe. The factor $\frac{R_{\text {lead }}}{R_{c}}=\frac{0,25}{1,3} \approx \frac{1}{5}$ originates from the resistivity of the lead from the heating power supply to the probe tip $R_{\text {lead }}=0,25(2) \Omega$ compared with the resistivity of the entire heating circuit $R_{c}=1,30(4) \Omega$ in cold state.

Fig. 5 shows the results of the inflection point method for all three EEP pins: The inflection points are only compared to the plasma parameters of the two cold Langmuir probes close to the EEP pins. In the low emission region, the inflection points of the emissive $I-V$ characteristics correspond well with the plasma potential values which result from the cold probe measurements, while underestimating them towards no emission. On the other hand a too high electron emission of the EEPs leads to an overestimation of the plasma potential values. This behaviour can be understood best as with a warm, but not hot, high emissive probe the space-charge effects in front of the probe are minimized, leading to the correct value of the plasma potential [22]. 

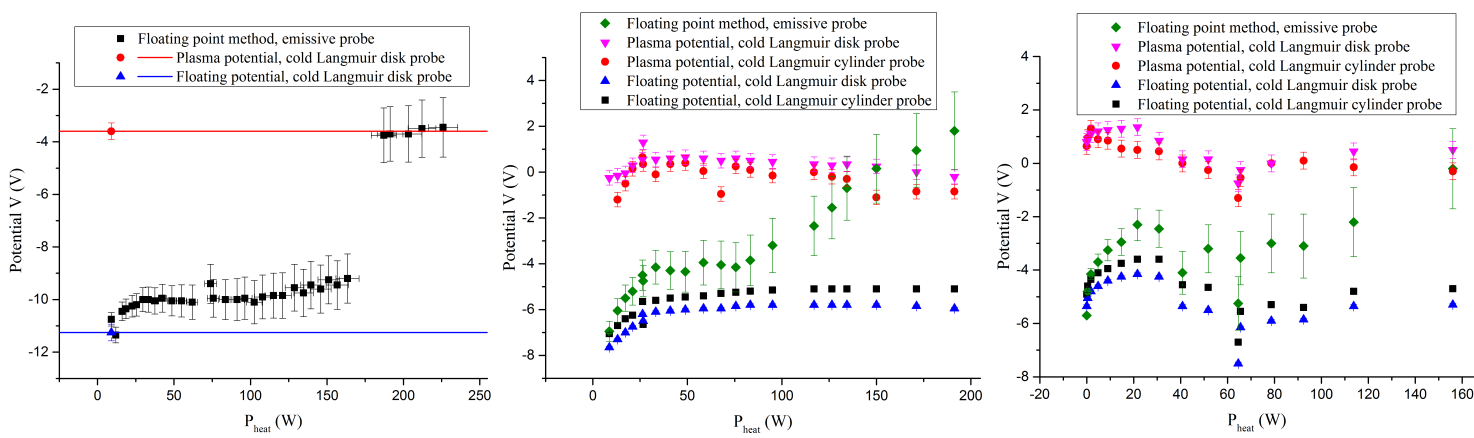

Figure 4: Comparing the floating potential $V_{f l}$ of the emissive $\mathrm{LaB}_{6}$ probe (left panel), TiC probe (centre panel) and pure $C$ probe (right panel) with the derived plasma potential of the two cold Langmuir probes.
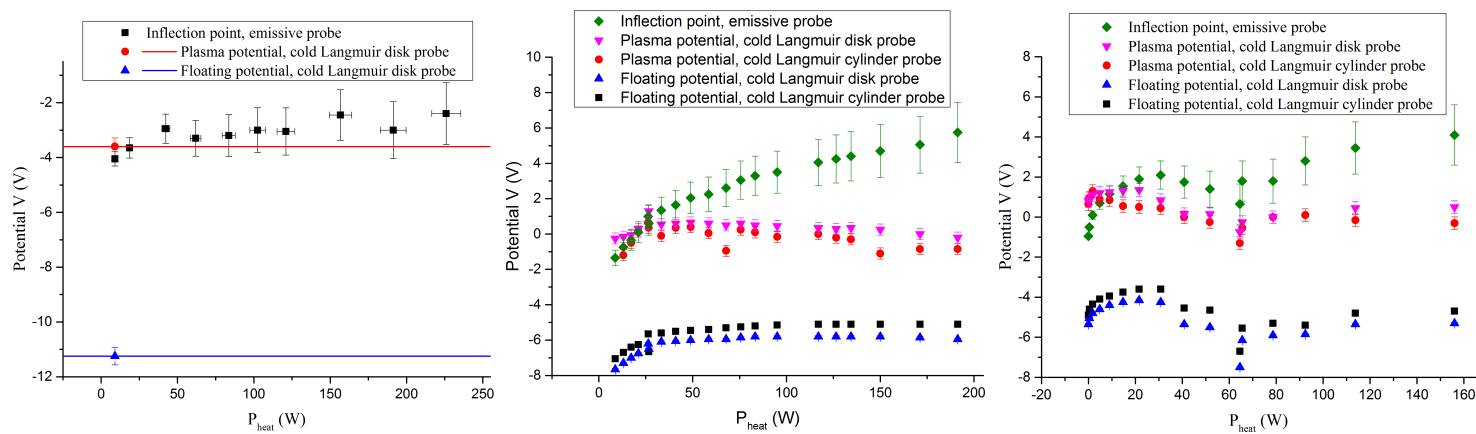

Figure 5: Using the inflection point method with an emissive $\mathrm{LaB}_{6}$ probe (left panel), TiC (centre panel) and pure $\mathrm{C}$ (right panel) pin to obtain the plasma potential.

\section{Conclusion}

It has been demonstrated that an ohmically heated $\mathrm{LaB}_{6}, \mathrm{TiC}$ and pure $\mathrm{C}$ probe tip can be used as an electron-emissive probe. The probe prototypes are capable of almost attaining the actual plasma potential in the chamber. Yet, there are still problems to heat a pure graphite rod to the necessary temperature for strong enough electron emission. The rather large uncertainties of the floating point technique indicated by the error bars in Fig. 4 and 5 resulting from the heating bias of the probes are greatly diminished when measuring in high and dense plasmas. There the additional passive heating by the plasma can be used to keep the probe in the working temperature regime during insertion and measurement time. This measurement technique strongly reduces measurement errors regarding electron temperature fluctuations and strongly reduces measurement time scales $(\mathrm{kHz}$ to $\mathrm{MHz})$ in fusion plasmas. These are vital attributes for measuring cross-field particle transport in tokamaks and stellarators.

\section{Acknowledgments}

This work has been carried out within the framework of the EUROfusion Consortium and has received funding from the Euratom research and training programme 2014-2018 under grant agreement No 633053. The views and opinions expressed herein do not necessarily reflect those of the European Commission. This work was also supported by the Friedrich Schiedel Foundation for Energy Technology and the Commission for the Coordination of Nuclear Fusion Research in Austria (Austrian Academy of Sciences). 


\section{References}

[1] I. Langmuir, The pressure effect and other phenomena in gaseous discharges, Journal of the Franklin Institute 196 751-762 (1923)

[2] H. M. Mott-Smith and Irving Langmuir, The theory of collectors in gaseous discharges, Physical Review 28727 (1926)

[3] R. L. Merlino, Understanding Langmuir probe current-voltage characteristics, Am. J. Phys. 751078 (2007)

[4] R. H. Huddlestone und Stanley L. Leonard, Plasma diagnostic techniques, Academic Press Inc., 133-200 (1965)

[5] I. H. Hutchinson, Principles of plasma diagnostics, Cambridge University Press (1987)

[6] J. P. Sheehan and N. Hershkowitz, Emissive probes, Plasma Sources Sci. Technol. 2022 (2011)

[7] A. A. Ovsyannikov and M. F. Zhukov, Plasma diagnostics, Cambridge International Science Publishing (2012)

[8] N. Hershkowitz et al., Selfemissive probes, Rev. Sci. Instrum. 5429 (1983)

[9] N. Hershkowitz et al., Emissive probe measurements of plasma potential fluctuations in the edge plasma regions of tokamaks, Review of Scientific Instruments 741583 (2003)

[10] R. Schrittwieser et al., Application of emissive probes for plasma potential measurements in fusion devices, Contrib. Plasma Phys. 41 494-503 (2001)

[11] R. W. Schrittwieser et al., A probe head for simultaneous measurements of electrostatic and magnetic Fluctuations in ASDEX Upgrade edge plasma, Contrib. Plasma Phys. 50860 - 865 (2010)

[12] M. Endler et al., Measurements and modelling of electrostatic Fluctuations in the scrape-off layer of ASDEX, Nuclear Fusion, Vol. 351307 (1995)

[13] C. Ionita et al., The use of emissive probes in laboratory and tokamak plasmas, Contrib. Plasma Phys. 51264 - 270 (2011)

[14] C. Ionita et al., Arrangement of emissive and cold probes for fluctuation and Reynolds stress measurements, Rev. Sci. Instrum. VOLUME 75 4331-4333 (2004)

[15] F. Cardarelli, Materials handbook, a concise desktop reference, Springer Verlag 553 (2008)

[16] H. F. Ivev, Thermionic electron emission from carbon, Phys. Rev. 76567 (1947)

[17] M. Bakr et al., Comparison of the heating properties of $L_{a B_{6}}$ and $\mathrm{CeB}_{6}$ due to the back bombardment effect in a thermionic RF gun Journal of the Korean Physical Society 59 3273-3279 (2011)

[18] P. Massacci, Proceedings of the XXI International Mineral Processing Congress, July 23-27 C3-57 (2000)

[19] Roempp Online - Rowmpp Lexikon Chemie. Georg Thieme Verlag, https://roempp.thieme.de/roempp4.0/do/data/RD-07-01808 (2011)

[20] A. Marek et al., Experimental investigation of the change of the electron saturation current of a dc-heated emissive probe, Czechoslovak Journal of Physics 56 B932-B937 (2006),

[21] A. Marek et al., Emissive probe diagnostic in low temperature plasma-effect of the space charge and variations of the electron saturation current, Contributions to Plasma Physics 48 491-496 (2008)

[22] J. R. Smith, N. Hershkowitz and P. Coakley, Inflection point method of interpreting emissive probe characteristics, Review of Scientific Instruments $\mathbf{5 0} 210$ (1979) 